\title{
La contaminación de los recursos hídricos como punto de partida para el activismo socio-científico
}

\author{
Teresa Conceição \\ Instituto de Educação da Universidade de Lisboa,Portugal.mariaconceicao@campus.ul.pt \\ ORCID: https:// orcid.org/0000-0002-5893-5509 \\ Mónica Baptista \\ Instituto de Educação da Universidade de Lisboa,Portugal.mbaptista@ie.ulisboa.pt \\ ORCID: bttps:// orcid.org/0000-0003-1609-5764 \\ Pedro Reis \\ Instituto de Educação da Universidade de Lisboa, Portugal.preis@ie.ulisboa.pt \\ ORCID: https:// orcid.org/0000-0002-9549-2516
}

[Recibido: 30 Enero 2018. Revisado: 29 Noviembre 2018. Aceptado: 3 Diciembre 2018]

\begin{abstract}
Resumen: Los currículos de ciencias destacan la necesidad de implicar al alumnado en temas de la sociedad civil relacionados con las ciencias, al poner en evidencia la primacía de los aprendizajes sobre la relación cienciastecnología-sociedad-ambiente. Este trabajo tiene como objetivo conocer los aprendizajes de los alumnos cuando se involucran en el intento de solución de la problemática relacionada con la contaminación del riachuelo aledaño a su escuela. La metodología de investigación es cualitativa, de orientación interpretativa y basada en la observación participante. Participan 21 alumnos de dos clases de $8 .^{\circ}$ nivel ${ }^{1}$ con un currículo alternativo que viven en un medio rural del sudoeste portugués. Los datos se recogieron a través del diario del profesor, documentos escritos y entrevistas a los alumnos (realizadas al final del estudio). Los resultados revelan que las vivencias positivas proporcionadas por el club de radio les dotaron de confianza y les incentivaron al activismo comunitario relacionado con la contaminación del riachuelo local. Este activismo se concreta a través de un teatro de fantoches sobre el tratamiento de las aguas residuales. Asimismo, los resultados nos muestran que el activismo conduce a los alumnos a la identificación de los temas de ciencias y de tecnología que están en la base de la contaminación del riachuelo, ampliando su conocimiento sobre el problema y discutiendo diferentes perspectivas para su solución. Además, los jóvenes reconocen que el conocimiento les permite informar a otros miembros de la comunidad y se dan cuenta de que tienen el derecho de implicarse en cuestiones socio-científicas que afecten su calidad de vida.
\end{abstract}

Palabras clave: Cuestiones socio-científicas; Problemáticas socioambientales en el aula; Alfabetización científica; Educación en ciencias.

Hidric resources pollution as a trigger for socio-scientific activim

\begin{abstract}
Science curricula emphasize the need to involve pupils in issues related with society, promoting the science-technology-society-environment perspective. The present study aims at understanding pupils' learning when they were involved in the problem related with the pollution of a creek near their school. In this study, a qualitative and interpretive methodology was used, based in participant observation. Participants are 21 pupils, who attend the 8th grade of an alternative curriculum. The school belongs to a rural area in the Southwest of Portugal. Data were collected through field notes, written documents and pupils' interviews (performed at the end of the study). The results show that the positive experiences provided by the radio club gave pupils confidence and encouraged them to activism related with the pollution of the local creek. This activism is concretized through a theater, raising awareness the community and local authority to the treatment of wastewater. In addition, the results show that activism allows pupils to indentify the scientific and technological concepts which are in the base of the pollution of the creek, expanding their knowledge related with the problem and discussing different perspectives for the solution. In addition, pupils recognize that is important to provide with knowledge the other members of the community and realize that they have the right to engage in socioscientific issues, that affects their quality of life.
\end{abstract}

Keywords: Collective activism on socio-scientific issues; Socio-scientific issues in the classroom; Scientific literacy; Science education.

${ }^{1}$ En el Sistema Educativo Español equivale al $2^{\circ}$ de la ESO.

Revista Eureka sobre Enseñanza y Divulgación de las Ciencias

Universidad de Cádiæ. APAC-Eureka. ISSN: 1697-011X

http:/ / dx.doi.org/10.25267/Rev_Eureka_ensen_divulg_cienc.2019.v16.i1.1502

http:/ / reuredc.uca.es 
Para citar este artículo: Conceição, T., Baptista, M., \& Reis, P. (2019) La contaminación de los recursos hídricos como punto de partida para el activismo socio-científico. Revista Eureka sobre Enseñanza y Divulgación de las Ciencias 16(1), 1502. doi: 10.25267/Rev_Eureka_ensen_divulg_cienc.2019.v16.11.1502

\section{Introducción}

Las ciencias y la tecnología tienen un gran impacto en los ecosistemas y en la calidad de vida de las poblaciones. Sin embargo, las comunidades no siempre tienen una participación activa y efectiva en la discusión y en la toma de decisiones sobre los temas relacionados con las ciencias y la tecnología. Esta problemática expone la necesidad de democratizar las ciencias, o sea, incluir en el debate público un conocimiento más plural, relacional y dialógico sobre las cuestiones socio-científicas (Colucci-Gray y Camino, 2014). De hecho, Little (2009) evidencia que es necesario conectar las comunidades locales, de un modo más efectivo, con las autoridades estatales que evalúan los riesgos de toxicidad ambiental. Según este autor, la comunidad posee un conocimiento que no siempre es tomado en igual consideración que la perspectiva de los científicos, por lo que es necesario construir alianzas más democráticas con las comunidades afectadas. Este déficit democrático en la toma de decisiones sobre cuestiones socio-científicas está bien expuesto por Goldstein (2017). En su estudio sobre la problemática de la central nuclear de Angra dos Reis, Brasil, la autora nos explica que las comunidades locales no son oídas y no están debidamente informadas sobre las consecuencias del proyecto. Según la autora, la situación se presenta más preocupante cuando se verifica que las poblaciones no tienen bien claro cómo actuar ante una situación de catástrofe. Estas problemáticas, a escala local y global, han tenido una fuerte influencia en los currículos de ciencias, siendo actualmente reconocida la importancia de la educación en ciencias en la toma de decisiones por parte de los alumnos en cuestiones socio-científicas (CSC) (Aikenhead, 2010; NRC, 2010; NGSS, 2013; Osborne y Dillon, 2008) y en la responsabilidad individual y cívica sobre las mismas (NAAEE, 2004).

La enseñanza de las ciencias a partir de CSC suscita el interés del alumnado por las ciencias (Freire, Faria, Galvão y Reis, 2013; Simon y Amos, 2011) y el aprendizaje de los conceptos científicos en diferentes áreas, por ejemplo, en Genética (Puig y Jiménez-Aleixandre, 2011), en Biología (Dawson, 2011), en Ciencias del Ambiente (Evagorou, 2011), en Química (Sadler, Klosterman, y Topcu, 2011) y en la Naturaleza de las Ciencias (Apotheker, Blonder, Akaygun, Reis, Kampschulte y Laherto, 2017; Reis y Galvão, 2004; Zeidler, Applebaum, y Sadler, 2011). Asimismo, otros autores nos dan a conocer que los alumnos, cuando están implicados en las CSC, amplían su conocimiento sobre la ética de las ciencias y se hacen más sensibles a estos temas (Eastwood, Schlegel, y Cook, 2011). Algunas de estas cuestiones dividen a la sociedad por el hecho de que existen explicaciones y soluciones basadas en principios morales no siempre conciliables (Stradling, 1985). Teniendo en cuenta la naturaleza y trascendencia de estas cuestiones por su elevado impacto en los ecosistemas y la falta de consenso en cuanto a las soluciones (e.g., Stawkowski, 2016), su discusión en clase conlleva diferentes interpretaciones de los hechos por parte de profesores y alumnos (Oulton, Dillon, y Grace, 2004). Por esta razón, la Enseñanza de las Ciencias a partir de la controversia socio-científica supone un reto para los profesores, pero también fomenta su desarrollo profesional. Por ejemplo, la desconfianza inicial detectada en Simon y Amos (2011) sobre la enseñanza de las ciencias a partir de las CSC es superada al final del estudio. Del mismo modo, Wong, Zeidler e Klosterman (2011) manifiestan alguna dificultad en planear las clases a partir de CSC, pero progresivamente ganan autonomía y acaban revelando entusiasmo en hacerlo. Algunos profesores temen que los alumnos no respondan bien, sin embargo, al final, quedan agradablemente sorprendidos por su desempeño y se muestran dispuestos a implementar esta estrategia de enseñanza (Zeidler, Bell, Sadler, y Eastwood, 2011). De hecho, la implicación de 
profesores en grupos de práctica de activismo socio-científico, creando materiales en conjunto, compartiendo y aprendiendo con las experiencias, constituye una forma de apoyo mutuo en la superación de obstáculos (Linhares y Reis, 2014; Reis, 2014a, b). Se entiende como activismo socio-científico, la participación democrática de los alumnos con el objetivo de intentar solucionar las CSC y atenuar las tensiones asociadas (Alsop y Bencze, 2014; Baptista, Reis y Andrade, 2018). Un aspecto importante en la capacitación de los alumnos sobre el activismo socio-científico es la implicación de la comunidad, una vez que se pretende ampliar la discusión de estas cuestiones más allá de la clase y del espacio escolar (Hodson, 2011; Reis, 2013). El fomento del activismo socio-científico en el contexto escolar es fundamental para que los alumnos sean ciudadanos de pleno derecho, lo que constituye una dimensión esencial de la alfabetización científica (Gray, Colucci-Gray, y Camino, 2009; Kolstø, 2001; Marques y Reis, 2017; Millar y Hunt, 2002; Reis, 2014a, b).

Partiendo de estas premisas, en este trabajo, se explora el activismo socio-científico con alumnos de $8^{\circ}$ nivel (entre los 13 y los 15 años). Más concretamente, se pretende conocer los aprendizajes de los alumnos cuando se involucran en el intento de solución de la problemática relacionada con la contaminación del riachuelo aledaño a su escuela. Este estudio ha sido desarrollado en el ámbito del Proyecto We Act-Promoting Collective Activism on Socio-Scientific Issues (Reis, 2014a, b).

\section{La cuestion socio-científica}

Muy cerca de la escuela pasa un riachuelo donde desaguan los efluentes domésticos sin el tratamiento apropiado. Como consecuencia, el riachuelo está contaminado, huele mal, por lo que el problema ambiental es evidente. Esta situación se agrava cuando no llueve. La comunidad conoce la situación y, naturalmente, muestran su malestar. Los vecinos se quejan del mal olor en sus casas que proviene de la cañería que lleva los efluentes hasta el riachuelo. El problema, además de ser antiguo, es político. De hecho, el Ayuntamiento ha prometido, en diversas campañas electorales, la solución del problema instalando una estación de aguas residuales. Sin embargo, el riachuelo continúa siendo un foco de contaminación ambiental y un riesgo para la salud pública. Así pues, se considera que esta cuestión tiene potencial para implicar los alumnos en el activismo socio-científico (Alsop y Bencze, 2014).

\section{Metodología}

\section{Participantes}

Participaron en este estudio 21 alumnos de $8^{\circ}$ nivel con edades comprendidas entre los 13 y los 15 años. Los alumnos pertenecían a dos clases con un currículo alternativo - elaborado específicamente para estos alumnos con necesidades específicas de aprendizaje- con áreas como horticultura, cocina y tecnologías de la información y comunicación. Estos jóvenes no mostraban interés por los temas escolares, no tenían hábitos de estudio, tenían un comportamiento poco adecuado y provenían de un medio rural desfavorecido.

\section{Método de recogida de datos}

Esta investigación es de naturaleza cualitativa (Erickson, 1986), según el paradigma de índole naturalista (Bogdan y Biklen, 1994), la cual se ha desarrollado durante un año lectivo. Los datos se han recogido por el método de observación participante. La investigadora ( $\mathrm{y}$ profesora de ciencias) participó en el proceso de aprendizaje de los alumnos, sacando notas en todas las clases. Los datos fueron complementados por entrevistas de tipo grupo focal realizadas a los alumnos al final de la investigación. Las entrevistas se centraron en las dimensiones: gusto/interés de los alumnos, dificultades y aprendizajes de los alumnos, percepciones sobre las clases de ciencias, activismo. Además, los alumnos realizaron ocho 
tareas durante las clases de ciencias, cuyos resultados fueron analizados para la investigación. Estas tareas fueron diseñadas por los autores según el modelo de los 5E de Bybee (1997). Este modelo se basa en una perspectiva constructivista del aprendizaje y contempla cinco fases: Envolver, Explorar, Explicar, Elaborar y Evaluar. En el envolvimiento se pretende captar el interés de los alumnos y su curiosidad por la investigación de un problema. En la fase de exploración, dirigida a la búsqueda de soluciones al problema, los alumnos plantearon y testaron hipótesis. En grupo, discutieron con los colegas los resultados y organizaron la información para comunicarla en la clase. En la fase de explicación, respondieron al problema, articulando lo que observaron con las hipótesis explicativas y su verificación. En esta fase, los alumnos fueron invitados a comunicar la solución del problema en la clase, usando un lenguaje científico y apoyándose en las evidencias recogidas. En la fase de elaboración, los alumnos aplicaron a otros contextos lo que aprendieron en la tarea, desarrollando competencias y ampliando conocimientos. Finalmente, en la fase de la evaluación, los alumnos reflexionaron sobre el trabajo desarrollado, evaluando la tarea y lo que aprendieron (Bybee, 1997). En esta investigación se realizarón ocho tareas distintas: La contaminación del arroyo; El ciclo del agua; El agua que usamos en la cocina; Aguas Residuales; Gestión sostenible del agua; Agricultura sostenible; Ciclo de los residuos biodegradables (de la huerta a la mesa y de la mesa hasta la huerta); y, Era una vez (los alumnos contaron sus experiencias en torno a la contaminación del agua de la ribera y pensaron en el modo de divulgarlas). Estas tareas pretendían capacitar los alumnos para (i) la comprensión de las fuentes de contaminación de los recursos hídricos y (ii) la acción centrada en resolución del problema específico de la contaminación del riachuelo (Hodson 2011; Reis 2013).

\section{Estructura y descripción de la actividad}

El activismo socio-científico, en esta investigación, se desarrolló en cinco etapas, según el modelo de Eilks (Sadler, Romine, y Topçu, 2016):

- Primera etapa. Identificación del problema y captación del interés del alumnado. La actividad es una salida de campo al riachuelo.

- Segunda etapa. Construcción del conocimiento científico necesario para comprender el problema y proponer soluciones. Las actividades consistieron en búsqueda de información sobre el funcionamiento de la estación de tratamiento de agua de consumo (ETA) y de la estación de tratamiento de aguas residuales (ETAR) y la preparación de un cuestionario para los técnicos de la ETA e ETAR en una visita de estudio.

- Tercera etapa. Identificación de soluciones, analizándose formas divergentes de poner en marcha la solución para buscar el consenso. Los alumnos percibieron las tensiones relacionadas con la contaminación del riachuelo a través de charlas informales con la población y con una actividad de role play que realizaron en clase. En esta actividad los alumnos representaron distintos personajes con intereses distintos relacionados con la solución del problema de polución, de manera que se hizo posible una discusión de ideas divergentes y el intento de encontrar consenso. $\mathrm{Al}$ final, escribieron un manifesto sobre la discusión generada en clase.

- Cuarta etapa. El activismo socio-científico. Los alumnos realizaron actividades relacionadas con la polución del riachuelo. Una de esas actividades fue la creación de un club de radio en la escuela. Los jóvenes visitaron una estación de radio donde entrevistaron a los técnicos para entender su funcionamiento, con preguntas preparadas por ellos. A continuación, crearon las normas de funcionamiento de la radio y se comprometieron a cumplirlas, esto con el apoyo de los profesores y con el 
conocimiento de la dirección. La radio funcionaba durante el recreo con la presencia de los profesores. Paralelamente, los alumnos trabajaron el activismo sobre la contaminación del riachuelo, con el creación de un teatro de fantoches para la comunidad.

- Quinta etapa, se hizo un balance de la actividad, con especial atención a los aprendizajes adquiridos, a las dificultades surgidas y a lo que agradó más o menos a los alumnos.

\section{Resultados}

Primera etapa. Durante la salida de campo, los alumnos advirtieron la existencia de un conducto abierto al riachuelo y observaron las condiciones en que se encontraba. Por ejemplo, uno de los alumnos comentó: En el riachuelo el agua está contaminada a causa del alcantarillado, el agua buele mal, está sucia y [tiene] peces muertos (registro escrito, tarea). El ejemplo muestra que los alumnos son sensibles a las señales de contaminación del riachuelo. De hecho, las observaciones evidencian un posible déficit de oxígeno disuelto en el agua y un tratamiento ineficaz de las aguas residuales. Luego, en clase se abordó el tratamiento de las aguas residuales y los alumnos se mostraron interesados, interviniendo de forma espontánea en la discusión. Sobre esto se puede citar una nota en el diario del profesor: Luisa dijo [en clase] que en la aldea donde vive hay una ETAR que no funciona. Los alumnos discuten entre ellos sobre la calidad del agua (riachuelos, ríos) de los lugares donde viven. Efectivamente, después de la salida de campo, el interés de los alumnos por una cuestión cotidiana hizo posible una discusión en clase con mucha participación sobre temas curriculares de las ciencias. Asimismo, durante la clase vino a colación el tema del tratamiento del agua de consumo humano y los alumnos revelaron un conocimiento superficial sobre el tema. Por ejemplo, a propósito del agua de consumo humano, Sebastião comentó: El pastor bebe agua de los charcos, dobla un paño varias veces y después chupa el agua. Ya lo bizo una vez para que mi padre lo viese y llenó una botella y ella estaba limpia (diario del profesor). Efectivamente, el alumno no parecía saber que el agua de consumo tiene que ser controlada bacteriológicamente y que existen tratamientos específicos. Las preguntas de los compañeros apoyan esta idea, pues Noel preguntó: ¿Por qué no se puede beber agua sucia?(registro escrito) y Ana quiso saber ¿Cómo se limpia el aguaé (registro escrito, tarea). Las cuestiones formuladas por los alumnos son adecuadas y revelan su interés y las ganas de ampliar sus conocimientos sobre el tema del tratamiento del agua de consumo. Por consiguiente, la profesora decidió, además, hacer una visita de estudio a la estación de tratamiento de agua de consumo (ETA). En lo que se refiere a la exploración del tema de la contaminación del riachuelo, los alumnos elaboraron guiones de entrevista a los técnicos de la ETAR y de la ETA, a partir de las búsquedas hechas en clase. Estas actividades proporcionaron a los alumnos oportunidades de identificar temas de ciencias relacionados con el problema de la contaminación del riachuelo. Por ejemplo, un grupo de alumnos preguntó: ¿Cómo se pueden ser tratar las aguas de la alcantarilla? ¿Cómo funciona una ETAR? ¿Por qué el agua de la alcantarilla desagua en el riachuelo? (registro escrito, tarea). En este ejemplo, se evidencia la curiosidad de los jóvenes por temas de su currículo que están relacionados con el problema de la contaminación del riachuelo. Además, a propósito de la ETA, otro grupo preguntó: ¿Qué nos hace el agua cuando está estropeada? ¿Cuáles son los productos para que el agua esté limpia y tratada? ¿Qué es mejor para el agua limpia, cloro u ozono? ¿Qué es más barato cloro $u$ ozono? (registro escrito, tarea). De hecho, estas cuestiones son relevantes en la vida cotidiana de los alumnos y de la comunidad. Además, son muy pertinentes en el contexto de la asignatura de las Ciencias Físicas y Naturales. Como resultado de toda esta curiosidad, los alumnos mostraron mucho interés en conocer el funcionamento de la ETA y la ETAR. Después de la elaboración de los guiones y de resueltos todos los trámites relacionados con la 
visita, ésta se realizó empezando por la ETA que se sitúa junto a una gran albufera y terminando en la ETAR.

Segunda etapa. Durante la visita guiada, los jóvenes hicieron las entrevistas a los técnicos y observaron todo, lo que favoreció que ampliaran sus conocimientos a este respecto. A este respecto, un grupo escribió: Aprendí como llega el agua a nuestras casas que es así, primero los señores consiguen que el agua llegue hasta la fábrica[s] suya y después la limpian, la tratan y después el agua viene para nuestras casa $[s]$ (registro escrito, tarea). En conjunto, los alumnos mostraron ampliar sus conocimientos sobre el recorrido del agua de consumo, desde que se recoge en la albufera, pasando por el tratamiento en la estación hasta las viviendas. Este tema, aun siendo paralelo al tema central, está relacionado y es importante para una comprensión más amplia de la problemática en estudio. Para que los alumnos comprendiesen el origen da la contaminación del riachuelo y discutiesen posibles soluciones, la visita a la ETAR ha sido especialmente relevante. Los datos evidencian que los alumnos reconocen la progresión de sus aprendizajes. Por ejemplo, uno de los grupos comenta: Aprendí como se trata el agua [de] consumo y la [de] la alcantarilla. Nosotros aprendimos como se trata el agua y para dónde va el agua de la alcantarilla (registro escrito, tarea). Es evidente que los alumnos adquirieron aprendizajes que son relevantes para la comprensión del problema en cuestión, particularmente sobre el tratamiento de las aguas residuales y su destino final. Es, precisamente, este conocimiento el que les permitirá ir más allá y comprender que el problema de la contaminación del riachuelo tiene solución a través de un adecuado tratamiento.

Tercera etapa. Después de la visita, en clase se volvió a la discusión sobre la problemática de la polución. Un grupo de alumnos identificó claramente la situación al comentar: Las aguas residuales no son tratadas y van para el riachuelo. (...) las aguas residuales son las que vienen de las casas de los vecinos y que van al riachuelo (registro escrito, tarea). Así, los alumnos se expresaron de forma adecuada sobre el riachuelo, revelando aprendizajes disciplinares importantes y una visión clara sobre el tema. A continuación, discutieron la localización de una hipotética ETAR en una tarea de role play en la cual representaron personajes con intereses y propuestas diferentes. En relación con esto, unos grupos comentaron:

Grupo 1- "El interés de la población del barrio XX es que la ETAR debería construirse en otro l ugar [no junto al riachuelo]. En el barrio quieren construir [en ese sitio] un parque verde".

Grupo 2 - "El interés del grupo [de protección] de las aves es defender la naturaleza" (registro escrito, tarea).

Otro grupo añadió:

"El grupo 3 defendió la empresa GP. Les conviene más la ETAR junto al riachuelo, una vez que proyectaron una urbanización en el terreno [destinado] a la [construcción] de la ETAR. El grupo 4 defendió la empresa YY. Les interesa construir la ETAR cerca del riachuelo, porque si no, no reciben contrapartidas de la empresa GP" (registro escrito, tarea).

De hecho, esta actividad, en la que los alumnos discutieron perspectivas diferentes, les permitió vislumbrar lo que podía estar detrás de los temas de base científica y tecnológica relacionados con el bienestar de la comunidad, adquiriendo un conocimiento más profundo y real sobre las CSC. Además, desarrollaron una perspectiva propia. Esto se hizo visible, por ejemplo, en la entrevista de grupo focal:

Profesora: En qué situación habéis sido incentivados a defender una opinión?

João - [en la tarea] de la ETAR.

Ana - [Queríamos] un jardin junto al riachuelo, las personas quieren pasear por allí y no pueden porque buele mal. 
En efecto, la discusión sobre la problemática de la contaminación del riachuelo, permitió que los alumnos dieran a conocer sus ideas y las defendieran. Además, el enfrentamiento de diferentes perspectivas representó una oportunidad para buscar buenos argumentos para fundamentar sus posiciones y reconocer los deberes y derechos de un ciudadano. En la evaluación de la actividad de role play los alumnos comentaron: Aprendemos a divulgar y a defender nuestros derechos. Aprendemos a debatir nuestros intereses. Como proteger nuestras cosas (registro escrito, tarea). Los alumnos-ciudadanos, al reconocer que tienen derechos en cuestiones públicas -como es el caso del tratamiento de las aguas residuales- y que estas cuestiones relacionadas con las ciencias, a veces, se codean con intereses ajenos, revelan aprendizajes importantes en el dominio del activismo socio-científico. Igualmente, valoran el conocimiento que les permite defender esos derechos. Así, al preguntar a los alumnos en la entrevista cómo adquirieron el poder de influir en las decisiones de otras personas en las cuestiones socio-científicas, Nelson contestó: Porque nosotros aprendemos y enseñamos a los demás. Rogério corroboró diciendo: Entonces si nosotros sabemos las cosas y la persona a la que bablamos tiene una opinión contraria le decimos lo que sabemos y ella después va a pensar en el tema, si es verdad, si no continua con la suya (entrevista de grupo focal).

En suma, la implicación de los alumnos en el activismo socio-científico sobre la problemática de la contaminación del riachuelo aledaño a la escuela, les llevó a aprender que las aguas residuales tienen que ser tratadas y que esto no se lleva a cabo en las aguas del conducto que desagua en el riachuelo. Además, les propició discutir posibles soluciones para el problema y reconocer que esas soluciones pueden afectar intereses ajenos. Además, reconocieron que tienen derechos "como miembros de la comunidad" en la discusión de los temas públicos de base científica y tecnológica y se percataron de que el conocimiento les permite influir en las opiniones de otros ciudadanos sobre controversias socio-científicas.

Cuarta etapa. En esta etapa, los jóvenes crearon los fantoches y escribieron el guion del teatro. A continuación, se trascribe un fragmento del guion y se ilustra un momento del teatro (figura 1).

Sebastião: ¿Entonces una ETA es apenas esto?

Sara: ¿Apenas, Sebastião? ¿Crees que tratar las aguas que llegan de nuestra casa es tarea fácil?

Margarida: Claro que no lo es, porque el agua pasa por varias fases en todo este proceso.

Carla: ¿Cuál es la diferencia entre una ETA y una ETAR, profesora Teresa?

Profesora: Después vamos a la ETAR y a partir de la visita guiada, tú misma vas a explicar la diferencia. ¿Puede ser?

Carla: ;Sí! jSí! Profesora jSeguro!

Noel: Entonces ¿Vamos a la ETAR?

María en "voz off': Ya en la ETAR.

Profesora: ;Ved, ved, chicos! Ya llegamos.

Sebastião: ¡Chiiì! ¿Qué olor!

Fernando: este no es olor a pies...jEs mucho peor!

Carla: ;Ya entendí cuál es la diferencia entre una ETA e una ETAR! Voy abora mismo a explicar todo a la profesora Teresa.

Noel: Mira, esta haciéndole la pelota a la profesora...jSerá que es tan difícil entender que una ETA trata el agua antes de llegar a nuestras casas y la ETAR lo hace después de salir de nuestras casas!

Fernando: ¡No sólo de nuestras casas, sabelotodo! 


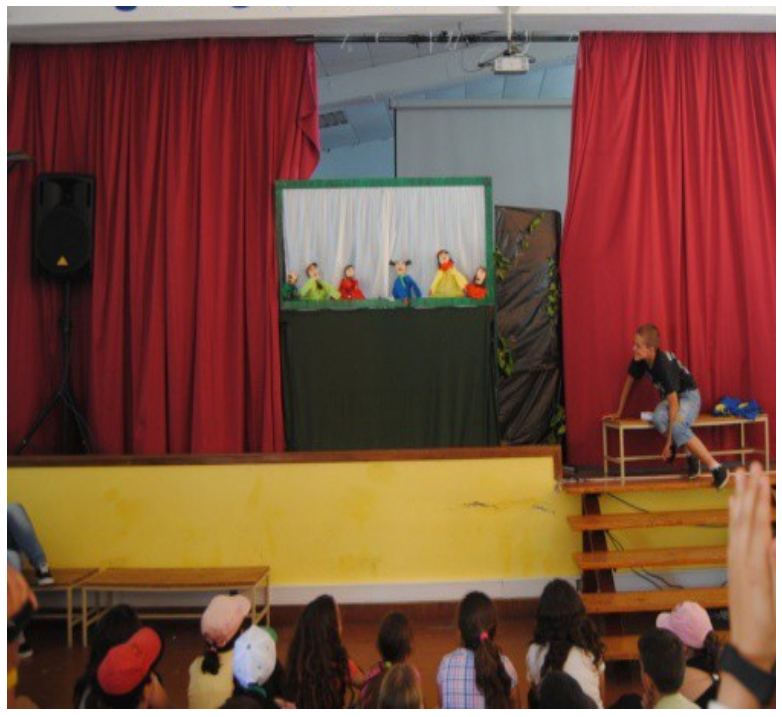

Figura 1. Teatro de fantoches.

En realidad, el activismo socio-científico realizado por los alumnos sobre la problemática del riachuelo local, discutiendo ampliamente los temas de ciencias y de tecnología que están en la base del problema y las posibles soluciones, bien como las responsabilidades individual, colectiva y política asociadas, llevó a los jóvenes a actuar fuera de clase. Esta iniciativa de los alumnos tuvo lugar en la escuela y pretendía ampliar sus conocimientos, tanto de su currículo como del dominio del activismo socio-científico y propiciar un acercamiento a toda la comunidad.

Por otro lado, la creación y dinamización de un club de radio, llevó a los alumnos a relacionarse con la comunidad, a través de un conjunto de actividades diversas, algunas relacionadas con su vocación profesional. Por ejemplo, en la visita de estudio a la radio local, los jóvenes participaron en un programa en directo, como puede indentificarse a continuación, en la figura 2.
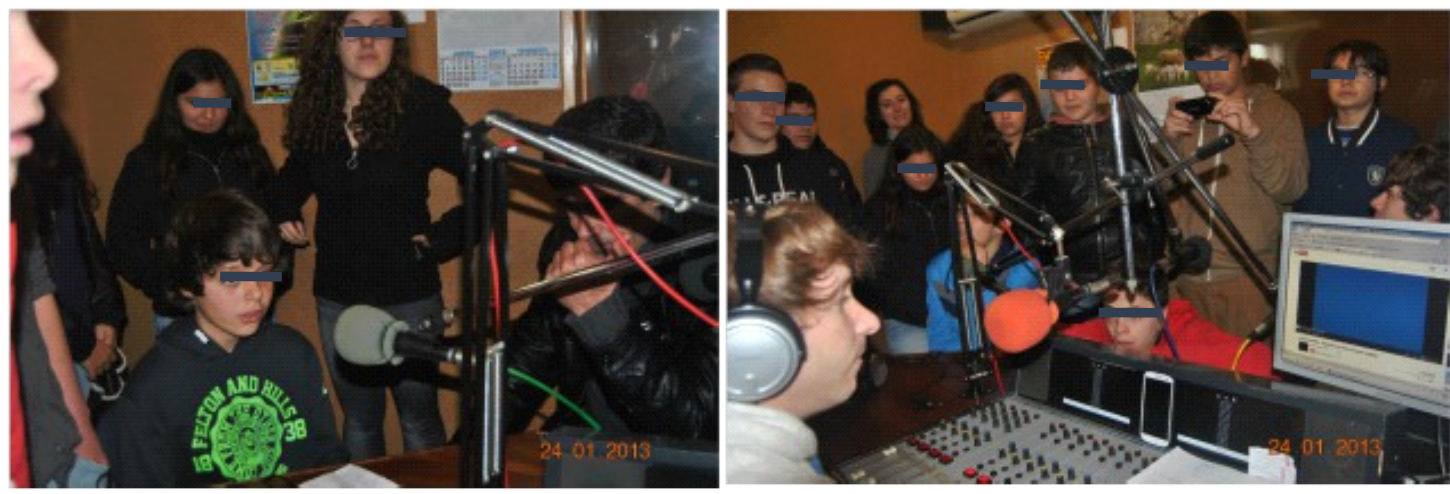

Figura 2. Visita de estudio a la radio.

Actividades como las relatadas, en las que los alumnos tuvieron la oportunidad de ver miembros de la comunidad desempeñando las profesiones que les gustan, los estimuló a participar en el programa de radio y los indujo a verse como ciudadanos. El cariz positivo de esta experiencia contribuyó a que ganasen confianza e interés por las actividades escolares. De vuelta a la escuela, los jóvenes sintieron ganas de crear un club de radio. Para ello, realizaron una serie de actividades importantes para su implicación en las actividades escolares, en 
particular en el activismo sobre la polución del riachuelo. Por ejemplo, para el funcionamiento del club, los alumnos crearon un horario de funcionamiento y una plantilla de programación. La creación del horario exigió la organización de los alumnos, según su tiempo libre y la disponibilidad de los profesores, y la asunción de la responsabilidad de trabajar en el recreo. Además, la elaboración de la plantilla de programación necesitó de gran esfuerzo de los alumnos: con lo que aprendieron en la estación de radio hicieron una búsqueda sobre los temas que podrían incluir en la programación y construyeron el formato de los programas. En el anexo 1, se presenta la plantilla de programación elaborada por los jóvenes.

La plantilla presenta una gran diversidad de programas, tanto informativos como lúdicos. De los temas presentados, el programa Canciones pedidas tuvo mucho éxito. En él, la audiencia podía escoger canciones y dedicárselas a alguien. Para ello, en un horario determinado, se recibían los pedidos en la estación y, después, se emitían.

De esta forma, los alumnos prestaban un servicio a toda la escuela, asumiendo responsabilidades como ciudadanos activos. El club de radio tuvo gran impacto en la escuela, ya que que animaba los recreos. Liderar este proyecto, proporcionó momentos de éxito y les hizo ganar confianza e interés para implicarse en el activismo.

Quinta etapa. Los alumnos hicieron un balance de las actividades que realizaron. En cuanto a lo que les gustó aprender, por ejemplo, respondieron "Me gustó saber lo que es una ETA y una ETAR", "Me gustó saber cómo se trata toda el agua", "Me gustó saber de dónde viene el agua de nuestras casas y, a donde va"," Lo que más me gustó fue ver la ETA y lo que menos me gustó fue el olor de la depuradora" (registro escrito, tarea). Estos ejemplos muestran bien que los alumnos se interesaron por investigar la cuestión socio-científica y que les gustó aprender contenidos disciplinarios relacionados. Esto es corroborado al mencionar lo que les gustaría aprender más. Por ejemplo, "Me gustaría saber todo sobre las riberas", "Me gustaría saber cómo se construyen las represas", "Me pregunto de dónde viene el mal olor del agua contaminada" (registro escrito, tarea). En realidad, a los alumnos les gustó profundizar la problemática de la contaminación del arroyo y mostraron interés por aprender más sobre este asunto.

\section{Conclusiones}

El activismo socio-científico sobre una cuestión del entorno relacionado con los alumnos hizo posible que se interesasen por ciencias y aprendiesen ciencias. Estos resultados están de acuerdo con otros estudios que también muestran que los alumnos aprenden ciencias cuando se implican en el activismo socio-científico (Alsop y Bencze, 2014; Baptista, Reis y Andrade, 2018; Blatt, 2013; Marques y Reis, 2017). Además, permitió a los alumnos implicarse en los temas públicos de la Ciencia, tomar decisiones y desarrollar su responsabilidad individual y cívica, tal como se aconseja en documentos internacionales sobre la Enseñanza de las Ciencias (NAAEE, 2004). Asimismo, al realizar activismo socio-científico, sobre todo cuando implica a la comunidad, los alumnos aprenden a verse como miembros de la comunidad, con deberes y derechos, tal como defienden otros autores (Colucci-Gray y Camino, 2014). Es más, mostraron un ejemplo de lo que es alfabetización científica (Gray et al., 2009; Hodson, 2011). En realidad, investigarón un problema de su comunidad, lo comprendieron a la luz del conocimiento científico y actuaron en el espacio escolar a través de un teatro de títeres, sugiriendo una solución al problema. Otro matiz del activismo socio-científico que hay que destacar es el hecho de que los alumnos aprendieron ciencias a partir de situaciones concretas con impacto social, discutiendo sus tensiones y la subjetividad y los procesos científicos asociados. Es justo este aprendizaje al que algunos autores se refieren como esencial para que el alumno pueda intervenir de manera informada en temas de ciencias y tecnologías (Driver, Leach, Millar, y Scott, 1996; Schwartz, Lederman, y Crawford, 2004). Así, el activismo socio- 
científico, tal como fue desarrollado en este investigación, supuso una mejora en la implicación de los alumnos para el aprendizaje de las ciencias y para su capacitación hacia una ciudadanía más participativa, aclarada y fundamentada (Hodson, 2011; Reis, 2014a,b). Es más, en esta investigación, los alumnos practicaron el activismo socio-científico más allá de la clase: organizando un teatro de fantoches para toda la comunidad para llamar la atención sobre el tratamiento de las aguas. El mensaje que se pretendía transmitir con esta iniciativa fue que la problemática de la contaminación del riachuelo era un tema socio-científico pero también político que creaba muchas tensiones en la comunidad local. La escuela no pretendía intensificar la tensión pero sí ayudar a suavizarla. Por consiguiente, una forma de sensibilizar a los miembros del Ayuntamiento sobre la urgencia de solucionar el problema fue a través de la representación de la obra de teatro que presentaba una solución, o sea, el tratamiento de las aguas, en general. En esta investigación, han sido varios factores los que contribuyeron para el éxito del activismo socio-científico sobre la contaminación del riachuelo: la relevancia de la problemática en la vida de los alumnos, la participación activa de los alumnos en su aprendizaje, la valoración de sus ideas en la discusión y la confianza que resultó de su capacitación para discutir cuestiones socio-científicas. El club de radio contribuyó a que los alumnos implicaran a la comunidad en el activismo, además, les proporcionó vivencias enriquecedoras, les permitió ganar el reconocimiento de sus compañeros y el resto de la comunidad. Progresivamente, han ido valorando cada vez más los temas escolares e implicando a la comunidad en el activismo. El activismo socio-científico debe ser un ejercicio de ciudadanía sobre temas públicos con base científica y tecnológica y debe servir para incentivar a los alumnos a actuar democráticamente y de una forma argumentada en los temas de ciencias con impacto en su vida y en la vida de la comunidad, tal como se ha incentivado en esta investigación.

\section{Referencias}

Aikenhead G. (2011) Towards a cultural view on quality science teaching. In D. Corrigan, J. Dillon, y R. Gunstone (Eds.) The Professional Knowledge Base of Science Teaching. Springer, Dordrecht. DOI.org/10.1007/978-90-481-3927-9_7

Alsop, S. y Bencze, L. (Eds.). Activism in science and technology education. London: Springer.

Apotheker, J., Blonder, R., Akaygun, S., Reis, P., Kampschulte, L. y Laherto, A. (2017). Responsible Research and Innovation in secondary school science classrooms: experiences from the project Irresistible. Pure and Applied Chemistry, 89(2), 211-219.

Baptista, M., Reis, P. y Andrade, V. (2018). Let's save the bees! An environmental activism initiative in elementary school. Visions for Sustainability, 9, 41-48.

Bogdan, R., y Biklen, S. (1994). Investigação qualitativa em educação: Uma introdução à teoria e aos métodos. Porto: Porto Editora.

Blatt, E. (2013). Local tree mapping: A collaborative, place-based activity integrating science, technology, math, and geography. Science Activities: Classroom Projects and Curriculum Ideas, 50(3), 99-109. doi:10.1080/00368121.2013.808165.

Bybee. R. W. (1997). Achieving scientific literacy: From purposes to practices. Portsmouth, NH: Heinemann.

Colucci-Gray, L., y Camino, E. (2014). From Knowledge to Action? Re-embedding Science Learning Within the Planet's Web. In S. Alsop \& L. Bencze (Eds.), Activism in science and technology education (pp. 149-164). London: Springer. 
Dawson, V. (2011). A case study of the impact ofi Socio-scientific issues into a reproduction unit in a catholic girls' school. In T. D. Sadler (Ed.), Socio-scientific Issues in the Classroom, (pp. 313-346). London: Springer.

Driver, R., Leach, J., Millar, R., y Scott, P. (1996). Young people's images of science. Buckingham: Open University Press.

Eastwood, J., Schlegel, W., y Cook, K. (2011). Effects of an interdisciplinary program on students' reasoning with socioscientific issues and perceptions of their learning experiences. In T. D. Sadler (Ed.), Socio-scientific Issues in the Classroom, (pp. 89-126). London: Springer.

Erickson, F. (1986). Qualitative methods in research on teaching. In M. C. Wittroch (Ed.), Handbook of research on teaching. New York, NY: Macmillan.

Evagorou, M. (2011). Discussing a socioscientific issue in a primary school classroom: The case of using a technology-supported environment in formal and nonformal settings. In T. D. Sadler (Ed.), Socio-scientific Issues in the Classroom, (pp. 133-160). London: Springer.

Freire, S., Faria, C., Galvão, C. y Reis, P. (2013). New curricular material for science classes: how do students evaluate it? Research in Science Education, 43, 163-178.

Goldstein, D. (2017). Fukushima in Brazil: undone science, technophilia, epistemic murk. Culture, Theory and Critique, 1-22. DOI: 10.1080/14735784.2017. 1357480

Gray, D., Colucci-Gray, L. y Camino, E. (Eds) (2009). Science, society and sustainability: Education and empowerment for an uncertain world. London: Routledge Research.

Hodson, D. (2011). Looking to the future. Building a curriculum for social activism. The Netherlands: Sense Publishers.

Kolstø, S. D. (2001). Scientific literacy for citizenship: Tools for dealing with the science dimension of controversial socio-scientific issues. Science Education, 85, 291-310.

Linhares, E. y Reis, P. (2014). La promotion de l'activism chez les futurs enseignants partant de discussion de questions socialement vives. Revue Francophone du Dévelopment Durable, 4, 80-93.

Little, P. (2009). Negotiating community engagement and science in the federal environmental public health sector. Medical Anthropology Quarterly, 23(2), 94-118. DOI: 10.1111/j.15481387.2009.01049.x

Marques, A. R., y Reis, P. (2017). Producción y difusión de vídeos digitales sobre contaminación ambiental. Estudio de caso: Activismo colectivo basado en la investigación. Revista Eureka sobre Enseñanza y Divulgación de las Ciencias 14(1), 215-226. Disponível em http://hdl.handle.net/10498/18857

Millar, R. y Hunt, A. (2002). Science for public understanding: A different way to teach and learn science. School science review, 83(304), 35-42.

NRC (2010). A framework for K-12 science education: Practices, crosscutting concepts, and core ideas. Committee on a conceptual framework for new K-12 science education standards. Board on science education, division of behavioral and social sciences and education. Washington, DC: The National Academies Press.

NGSS (2013). Next Generation Science Standards: For states, by states. Washington, DC: The National Academies Press. 
[NAAEE] North American Association for Environmental Education. (2004). Excellence in environmental education: Guidelines for learning (Pre-K-12). Available at http://www.naaee.org/npeee/learner_guidelines.php

Oulton, C., Dillon, J., y Grace, M. (2004). Reconceptualizing the teaching of controversial issues. International Journal of Science Education, 26(4), 411-423.

Osborne, J. y Dillon, J. (2008). Science education in Europe: Critical reflections. A report to the Nuffield Foundation. London: King's College London.

Puig. B., y Jiménez-Aleixandre, M. P. (2011). Different music to the same score: Teaching about genes, environment, and human performances. In T. D. Sadler (Ed.), Socio-scientific Issues in the Classroom, (pp. 201-238). London: Springer.

Reis, P. (2014a). Promoting students' collective socio-scientific activism: Teacher's perspectives. In S. Alsop \& L. Bencze (Eds.), Activism in science and technology education, (pp. 547-574). London: Springer.

Reis P. (2014b). Acción socio-política sobre cuestiones socio-científicas: reconstruyendo la formación docente y el currículo. Uni-Pluri/versidad, 14(2), 16-26. Disponível em: http://aprendeenlinea.udea.edu.co/revistas/index.php/unip

Reis, P. (2013). Da discussão à ação sócio-política sobre controvérsias sócio-científicas: uma questão de cidadania. Ensino de Ciências e Tecnologia em Revista, 3(1), 1-10.

Reis, P. y Galvão, C. (2004). Socio-scientific controversies and students' conceptions about scientists. International Journal of Science Education, 26(13), 1621-1633.

Schwartz, R.S., Lederman, N., y Crawford, B. (2004). Developing views of nature of science in an authentic context: An explicit approach to bridging the gap between nature of science and scientific inquiry. Science education 88(4), 610-645.

Sadler, T. (2011). Socio-scientific issues-based education: What we know about science education in the context of SSI. In T. D. Sadler (Ed.), Socio-scientific Issues in the Classroom, (pp. 355-369). London: Springer.

Sadler, T., Klosterman, M., y Topcu, M. (2011). Learning science content and socio-scientific reasoning through classroom explorations of global climate change. In T. D. Sadler (Ed.), Socio-scientific Issues in the Classroom, (pp. 45-78). London: Springer.

Simon, S., y Amos, R. (2011). Decision making and use of evidence in a socio-scientific problem on air quality. In T. D. Sadler (Ed.), Socio-scientific Issues in the Classroom, (pp. 167192). London: Springer.

Stawkowski, M. (2016). "I am a radioactive mutant": Emergent biological subjectivities at Kazakhstan's Semipalatinsk nuclear test site. American Ethnologist, 43(1), 144-157. DOI: 10.1111/amet.12269.

Stradling, R. (1985). Controversial issues in the curriculum. Bulletin of Environmental Education, 170, 9-13.

Wong, S., Zeidler, D., y Klosterman, M. (2011). Metalogue: Preconditions and resources for productive socio-scientific issues teaching and learning. In T. D. Sadler (Ed.), Socioscientific Issues in the Classroom, (pp. 271-276). London: Springer.

Zeidler, D., Applebaum, S., y Sadler, T. (2011). Enacting a socioscientific issues classroom: Transformative transformations. In T. D. Sadler (Ed.), Socio-scientific Issues in the Classroom, (pp. 277-306). London: Springer. 
Anexo 1. Plantilla de programación de radio elaborada por los jóvenes

\begin{tabular}{|c|c|}
\hline \multicolumn{2}{|c|}{ PLANTILLA DE PROGRAMACIÓN } \\
\hline$\underline{9: 00}$ & $\underline{\text { Meteorología }}$ \\
\hline$\underline{9: 10}$ & $\underline{\text { Música portuguesa }}$ \\
\hline$\underline{9: 35}$ & $\underline{\text { Canciones pedidas }}$ \\
\hline$\underline{10: 00}$ & $\underline{\text { Entrevista }}$ \\
\hline$\underline{10: 15}$ & $\underline{\text { Música }}$ \\
\hline$\underline{10: 40}$ & $\underline{\text { Videoclips }}$ \\
\hline$\underline{11: 00}$ & $\underline{\text { Canciones pedidas }}$ \\
\hline$\underline{11: 10}$ & $\underline{\text { Pasatiempo }}$ \\
\hline$\underline{11: 25}$ & $\underline{\text { Respuesta al pasatiempo }}$ \\
\hline$\underline{11: 30}$ & $\underline{\text { Entrega de premios }}$ \\
\hline$\underline{11: 40}$ & $\underline{\text { Pausa }}$ \\
\hline$\underline{12: 10}$ & $\underline{\text { Música }}$ \\
\hline$\underline{12: 30}$ & $\underline{\text { Comida/Notícias sobre temas relevantes para }}$ \\
\hline$\underline{14: 00}$ & $\underline{\text { la comunidad }}$ \\
\hline$\underline{14: 30}$ & $\underline{\text { Música }}$ \\
\hline$\underline{14: 50}$ & $\underline{\text { Música portuguesa }}$ \\
\hline$\underline{15: 00}$ & $\underline{\text { Películas y músicas }}$ \\
\hline$\underline{15: 50}$ & $\underline{\text { Chistes }}$ \\
\hline
\end{tabular}

